\title{
Study of current leakage in InAs $p-i-n$ photodetectors
}

\author{
Ray-Ming Lin ${ }^{\text {a) }}$ \\ Department of Electronic Engineering, Chang Gung University, Tao-Yuan, Taiwan, Republic of China \\ Shiang-Feng Tang and C. H. Kuan \\ Department of Electrical Engineering, National Taiwan University, Taipei, Taiwan, Republic of China
}

(Received 18 February 2000; accepted 28 August 2000)

\begin{abstract}
The current leakages of InAs photodiodes have been systematically studied by adding undoped layers having thicknesses of $0,0.30$, and $0.72 \mu \mathrm{m}$ between the $p-n$ junction. At reverse bias $V$ $=-0.5 \mathrm{~V}$, the dark currents of the InAs $p-i-n$ diodes with undoped layer thicknesses of $0,0.30$, and $0.72 \mu \mathrm{m}$ are about $5 \times 10^{-6}, 7 \times 10^{-8}$, and $1 \times 10^{-10} \mathrm{~A}$, respectively, at $77 \mathrm{~K}$. The leakage current of the InAs $p-n$ diode was successfully reduced by adding $0.72-\mu \mathrm{m}$-thick undoped layer between the $p-n$ junction. (C) 2000 American Vacuum Society. [S0734-211X(00)04406-1]
\end{abstract}

\section{INTRODUCTION}

InAs infrared photodetectors and infrared (IR) lightemitting diodes are useful for applications that use 3-5 $\mu \mathrm{m}$ atmospheric windows. ${ }^{1-3}$ Compared to other narrow-band gap III-V semiconductor materials such as antimonide compounds, the prime advantage of InAs is that it can be operated at room temperature. ${ }^{4,5}$ This is very important because the cryogenic cooling system, necessary for devices operated at low temperature, makes the conventional IR devices bulky and inconvenient in mobile use. Thus, InAs diodes are more suitable in use as room-temperature-operated IR detectors, i.e., environmental protection for hazardous gas detection. ${ }^{6-8}$

In general, photodiodes made of narrow-band gap materials are operated under zero bias because of their high leakage current and low breakdown voltage. Infrared diodes are selected according to the following requirement: the zero-bias differential resistance and area product $\left(R_{0} A\right)$ should be as high as possible. ${ }^{9}$ However, the $R_{0} A$ product is degraded by an increase in current leakage. Thus, there is interest in understanding the leakage current mechanisms of photodiodes made of narrow-band gap materials, so as to minimize this current. For InSb and InAs, the surface states due to material or process-induced defects may cause a serious shunt leakage current $^{9,10}$ and may increase excess noise, which greatly degrades the detector sensitivity. For the diode without a passivation layer on the surface, an inversion layer is formed on the surface of the $p$-type InAs layer. The bulk Fermi level penetrates into the conduction band at the surface inversion layer, and the quantized subbands are populated with electrons. This inversion layer, formed in the $p$ region, will be in ohmic contact with the nearby $n$-type substrate with an accumulated surface. ${ }^{11}$ A lateral $p-n$ tunnel diode is formed at the side wall of the $p$-region surface. Two conditions must be met for the tunneling to occur: first, the bottom of the conduction band at the surface must overlap in energy with the top of the valence band within the bulk. Second, the doping level of the bulk must be sufficiently high so that the width of the depletion region separating the conduction band in the surface inversion layer from the bulk valence band is narrow

${ }^{a)}$ Electronic mail: rmlin@mail.cgu.edu.tw enough to yield an appreciable tunneling probability. When applying reverse bias, the electrons in the valence band of the $p^{+}$layer can tunnel into the surface $n^{+}$subband and form a leakage path through the $n$ region. That is the reason why the unpassivated InAs $p-n$ diode has a large reverse leakage current. So, it is believed that the diode needs an optimal passivation treatment or must include a gatecontrolled diode design to get a leak-free diode. ${ }^{11-14}$

In this work, we have systematically studied the current leakage of the unpassivated InAs $p-i-n$ diodes by varying the undoped layer thickness of $0,0.30$, and $0.72 \mu \mathrm{m}$. In addition, the temperature-dependent detectivity $\left(D^{*}\right)$ of these InAs $p-i-n$ photodetectors are also studied in detail.

\section{EXPERIMENTS}

An InAs diode was grown on (100) $n$-type InAs substrates using a VG V80H MK II solid-source molecular-beam epitaxy (MBE) unit equipped with a $15 \mathrm{kV}$ reflection highenergy electron diffraction system. After loading the substrate into the growth chamber, they were slowly heated to $510{ }^{\circ} \mathrm{C}$ to remove surface oxide, then a $0.2-\mu \mathrm{m}$-thick $n$-type InAs buffer layer (Si doped to $1 \times 10^{18} \mathrm{~cm}^{-3}$ ) was grown to smooth out the surface. Next a $1-\mu \mathrm{m}$-thick $n$-type InAs active layer (Si doped to $5 \times 10^{16} \mathrm{~cm}^{-3}$ ) was deposited, followed by an undoped InAs layer with various thickness, i.e., $0,0.30$, and $0.72 \mu \mathrm{m}$. The doping level in the $i$ layer represents the background doping level in the MBE system. Subsequently, a $0.1-\mu \mathrm{m}$-thick $p$-type InAs layer (Be doped to $1 \times 10^{18} \mathrm{~cm}^{-3}$ ) was grown, and finally, a $0.1-\mu \mathrm{m}$-thick $p$-type InAs contact layer (exponentially graded from 1 $\times 10^{18}$ to $1 \times 10^{19} \mathrm{~cm}^{-3}$ ) was deposited. The growth rate was kept at $1 \mu \mathrm{m} / \mathrm{h}$. The $\mathrm{As}_{4} / \mathrm{III}$ beam equivalent pressure ratio was maintained at about 25 throughout the growth.

To fabricate the InAs photodiode, the epilayers are first mesa etched into circular dots $200 \mu \mathrm{m}$ in diameter. A double layer of 100-nm-thick $\mathrm{Au}-\mathrm{Be}$ and 300-nm-thick $\mathrm{Au}$ was evaporated and lifted off sequentially to form a $p$-type ohmic contact. The $n$-type ohmic contact was formed by In mounted during MBE. The detailed growth and fabrication procedures of the InAs $p-i-n$ diode are described in our previous article. 


\section{RESULTS AND DISCUSSION}

The semilog scale current-voltage $(I-V)$ characteristics of three typical unpassivated $p-i-n$ diodes with an undoped layer (i-layer) thickness $(d)$ of $d=0,0.30$, and $0.72 \mu \mathrm{m}$ between the $p-n$ junction are shown in Fig. 1(a) $(d=0 \mu \mathrm{m})$, Fig. 1(b) $(d=0.30 \mu \mathrm{m})$, and Fig. 1(c) $(d=0.72 \mu \mathrm{m})$ in the temperature range from 40 to $300 \mathrm{~K}$. At reverse bias $V$ $=-0.5 \mathrm{~V}$, the dark current of the InAs $p-i-n$ diodes with undoped layers of $0,0.30$, and $0.72 \mu \mathrm{m}$ are about 5 $\times 10^{-6}, 7 \times 10^{-8}$ and $10^{-10} \mathrm{~A}$, respectively, at $77 \mathrm{~K}$, and $1.2 \times 10^{-4}, 4.0 \times 10^{-5}$, and $6.0 \times 10^{-5} \mathrm{~A}$, respectively, at 300 K. In Fig. 1(a), a small tunneling peak occurs in the forward bias when the temperature is below $80 \mathrm{~K}$, indicating the formation of a surface leakage channel. ${ }^{15}$ However, tunneling peaks are not observed in the InAs $p-i-n$ diodes with undoped layer thicknesses beyond $0.3 \mu \mathrm{m}$. This suggests that the insertion of an undoped layer between the $p-n$ junction reduces the surface pinning effect. In addition, at $77 \mathrm{~K}$, the dark currents of the two unpassivated $p-i-n$ samples, with $i$-layer thicknesses of 0.30 and $0.72 \mu \mathrm{m}$, are both disturbed by the background thermal radiation.

In Fig. 1(a), the tunneling current is dominant below 80 $\mathrm{K}$. This can be explained by using the tunneling effect in the lateral $p^{+}-n^{+}$junction as follows: below $80 \mathrm{~K}$, the valenceband density of state $N_{v}$ in the region is nearly $10^{17} \mathrm{~cm}^{-3}$, and the doping of the $p$ layer is $1.0 \times 10^{18} \mathrm{~cm}^{-3}$, which is degenerate in this temperature range. Also, the current level of the InAs $p-i-n$ diode with a $0.72-\mu$ m-thick $i$ layer is almost four orders of magnitude lower than the above value of the InAs $p-n$ diode at the same reverse bias. Thus, the existence of an undoped layer more than $0.3 \mu \mathrm{m}$ thick between the InAs $p-n$ junction is sufficient to suppress the tunneling current. In addition, the reverse dark current of the unpassivated $p-i-n$ diode with a $0.72-\mu \mathrm{m}$-thick undoped layer becomes nearly independent of reverse biases at the temperature range of $40-300 \mathrm{~K}$.

The $R_{0} A$ versus reciprocal temperature plots of InAs $p-i-n$ diodes with $i$-layer thicknesses $(d)$ of $0,0.30$, and 0.72 $\mu \mathrm{m}$ are shown in Fig. 2. A steep drop in $R_{0} A$ value is observed at $160 \mathrm{~K}$. Above $160 \mathrm{~K}$, the activation energies $(\Delta E)$, extracted from the slope of the plots in Fig. 2, are about $0.3349(d=0 \mu \mathrm{m}), 0.3320(d=0.30 \mu \mathrm{m})$, and $0.3327(d$ $=0.72 \mu \mathrm{m}) \mathrm{eV}$. We found that the $\Delta E$ of the three samples are all nearly equal to the band gap of InAs, and it can be said that the bulk diffusion current dominates the transport mechanism in the temperature range. Below $160 \mathrm{~K}$, the activation energies of InAs $p-i-n$ diodes with $i$-layer thicknesses of $0,0.30$, and $0.72 \mu \mathrm{m}$ drop to about 2.8, 2.8, and 9.1 $\mathrm{meV}$, respectively, which are much lower than the band gap of InAs. This implies that the high lateral leakage current are dominant in the InAs $p-n$ diode, but only part of the surface leakage current is responsible for the samples with an undoped layer of 0.30 and $0.72 \mu \mathrm{m}$ between the $p-n$ junction. So, the existence of an undoped layer between the InAs $p-n$ junction could somehow effectively reduce the diode surface leakage tunneling current.

When tested under a $500 \mathrm{~K}$ blackbody source, the specific
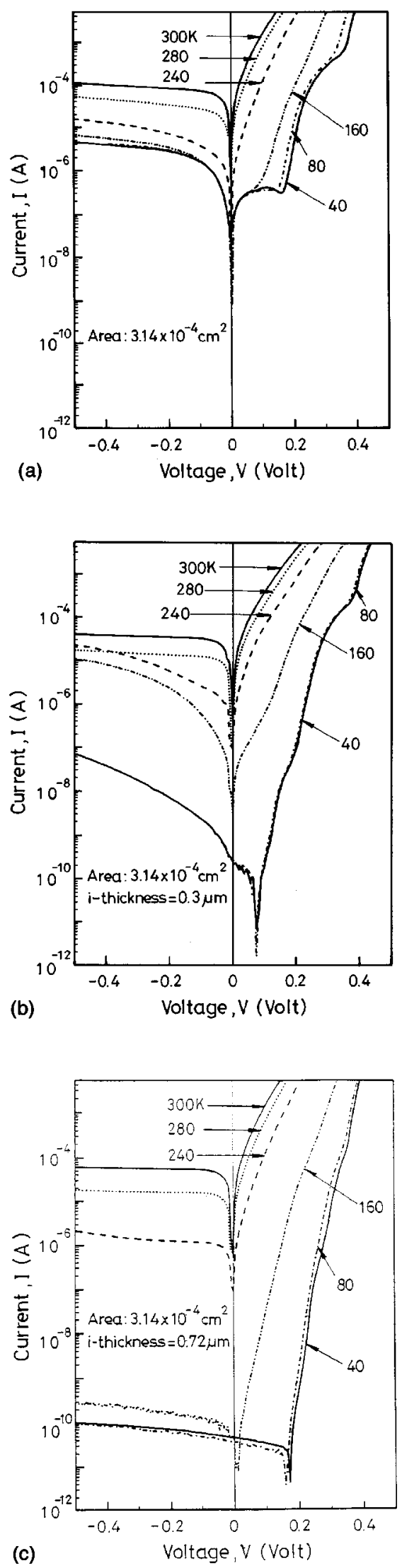

FIG. 1. Temperature-dependent semilog $I-V$ characteristics of the unpassivated InAs $p-i-n$ diodes with an $i$-layer thickness (d) of (a) $d=0$, (b) $d$ $=0.3 \mu \mathrm{m}$, and (c) $d=0.72 \mu \mathrm{m}$. 


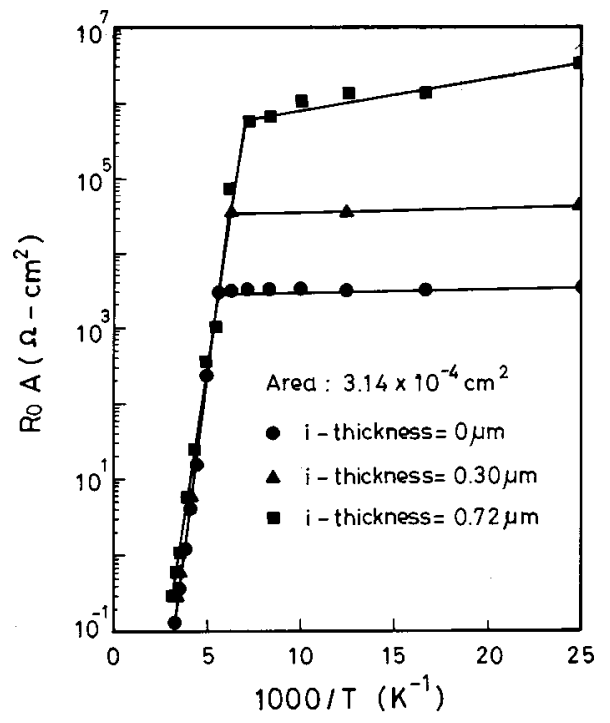

FIG. 2. $R_{0} A$ vs reciprocal temperature plot of the unpassivated InAs $p-i-n$ diodes with different $i$-layer thicknesses.

detectivity of the three InAs $p-i-n$ photodiodes with different $i$-layer thicknesses, i.e., $0,0.30$, and $0.72 \mu \mathrm{m}$, were measured and are shown in Fig. 3. At $77 \mathrm{~K}$, the specific detectivities of the InAs $p-i-n$ photodiodes with $0,0.30$, and $0.72-\mu \mathrm{m}$-thick $i$ layers are about $1.0 \times 10^{11}, 3.2 \times 10^{11}$, and

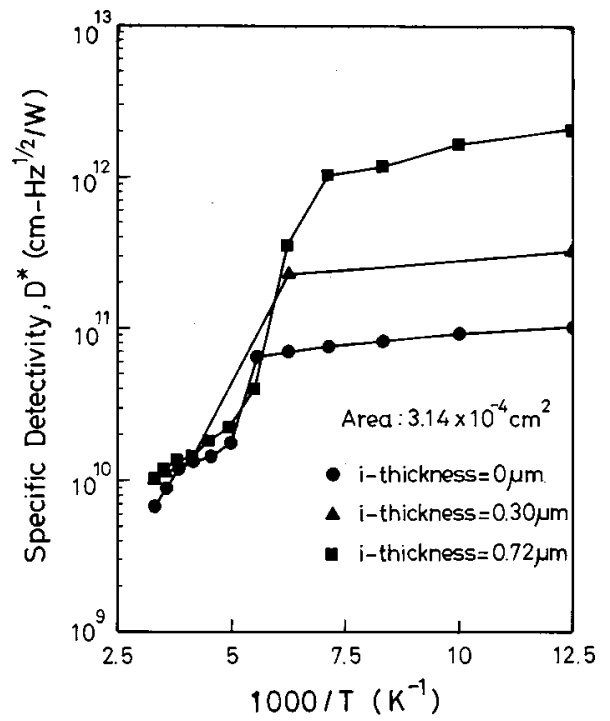

FIG. 3. Specific detectivity $\left(D^{*}\right)$ vs reciprocal temperature plot of the unpassivated InAs $p-i-n$ diodes with different $i$-layer thicknesses.
$2.1 \times 10^{12} \mathrm{~cm} \mathrm{~Hz}^{1 / 2} / \mathrm{W}$, respectively. When the temperature is greater than $160 \mathrm{~K}$, the specific detectivities of the InAs photodiodes with $0,0.30$, and $0.72-\mu \mathrm{m}$-thick $i$ layers all decrease rapidly, down to $6.2 \times 10^{9}, 9.2 \times 10^{9}$, and 1.2 $\times 10^{10} \mathrm{~cm} \mathrm{~Hz}^{1 / 2} / \mathrm{W}$, respectively, at room temperature. The results also strongly support the previous conclusion on the changes of $R_{0} A$ versus reciprocal temperature.

\section{CONCLUSION}

Current leakages of the unpassivated InAs $p-i-n$ diodes with undoped layer thicknesses of $0,0.30$, and $0.72 \mu \mathrm{m}$ have been systematically studied. At reverse bias $V=-0.5 \mathrm{~V}$, the dark currents of InAs $p-i-n$ diodes with undoped layers of $0,0.30$, and $0.72 \mu \mathrm{m}$ are about $5 \times 10^{-6}, 7 \times 10^{-8}$, and $10^{-10} \mathrm{~A}$, respectively at $77 \mathrm{~K}$. The leakage current of the InAs $p-n$ diode was successfully reduced by adding $0.72-$ $\mu \mathrm{m}$-thick undoped InAs layer between the $p-n$ junction. When tested under a $500 \mathrm{~K}$ blackbody source, the specific detectivities of the $p-i-n$ photodiodes with undoped layer thicknesses of $0,0.30$, and $0.72 \mu \mathrm{m}$ are $1.0 \times 10^{11}, 3.2$ $\times 10^{11}$, and $2.1 \times 10^{12} \mathrm{~cm} \mathrm{~Hz}^{1 / 2} / \mathrm{W}$, respectively, at $77 \mathrm{~K}$. Above $160 \mathrm{~K}$, the specific detectivities of the InAs photodiodes with $0,0.30$, and $0.72-\mu \mathrm{m}$-thick $i$ layers all decrease rapidly, down to $6.2 \times 10^{9}, 9.2 \times 10^{9}$, and $1.2 \times 10^{10} \mathrm{~cm}$ $\mathrm{Hz}^{1 / 2} / \mathrm{W}$, respectively, at room temperature.

\section{ACKNOWLEDGMENTS}

The authors are thankful for technical assistance from Professor Si-Chen Lee and Hao-Hsiung Lin. This work was supported by the National Science Council of the Republic of China under Contract No. NSC 89-2215-E-182-005.

${ }^{1}$ M. K. Parry and A. Krier, Electron. Lett. 30, 1968 (1994).

${ }^{2}$ W. Dobbelaere, J. DeBoeck, P. Hermans, R. Mertens, G. Borghs, W. Luyten, and J. Van Landuyt, Appl. Phys. Lett. 60, 868 (1992).

${ }^{3}$ W. Dobbelaere, W. De Raedt, J. De Boeck, R. Mertens, and G. Borghs, Electron. Lett. 28, 372 (1992).

${ }^{4}$ H. Fujisada and T. Sasase, Jpn. J. Appl. Phys., Part 1 23, 1162 (1984).

${ }^{5}$ R. M. Lin, S. F. Tang, S. C. Lee, C. H. Kuan, G. S. Chen, T. P. Sun, and J. C. Wu, IEEE Trans. Electron Devices 44, 209 (1997).

${ }^{6}$ M. K. Parry and A. Krier, Semicond. Sci. Technol. 18, 1764 (1993).

${ }^{7}$ A. Krier, Appl. Phys. Lett. 56, 2428 (1990).

${ }^{8}$ C. G. Bailey, M. Y. Yen, B. F. Levine, K. K. Choi, and A. Y. Cho, Appl. Phys. Lett. 51, 1431 (1987).

${ }^{9}$ S. Maniv, Proc. SPIE 819, 103 (1987)

${ }^{10}$ I. Bloom and Y. Nemirovsky, IEEE Trans. Electron Devices 40, 309 (1993).

${ }^{11}$ S. Margalit, J. Shappir, and I. Kidron, J. Appl. Phys. 46, 3999 (1975).

${ }^{12}$ Y. Nemirovsky, R. Adar, A. Kronfeld, and I. Kidrin, J. Vac. Sci. Technol. A 4, 1986 (1986)

${ }^{13}$ S. Margalit, J. Shappir, and I. Kidron, Solid-State Electron. 30, 1289 (1987).

${ }^{14}$ A. K Sood and S. P. Tobin, IEEE Electron Device Lett. 1, 12 (1980).

${ }^{15}$ L. Esaki, IEEE Trans. Electron Devices 23, 644 (1976). 